\title{
La gestión del conocimiento en empresas de comercio minorista de bienes en Villa Clara, Cuba
}

\author{
Raúl Yoel La Fé Jiménez* \\ Facultad de Ciencias Económicas \\ Universidad Central "Marta Abreu" de Las Villas
}

\section{Resumen}

En este ensayo se elabora un modelo de gestión del conocimiento para empresas del comercio minorista de bienes en Villa Clara. Se parte de una amplia revisión bibliográfica acerca de la gestión del conocimiento y mediante un análisis empírico se estudia la necesidad de implementarla en este tipo de empresas. Finalmente, se propone el modelo a partir de instrumentos de análisis que contribuyen a su conformación y es validado positivamente por expertos de la provincia de Villa Clara.

\section{Palabras clave}

Comercio, conocimiento, gestión, minorista, modelo. 


\title{
Knowledge management in the goods retail sector in Villa Clara, Cuba
}

\author{
Raúl Yoel La Fé Jiménez* \\ Facultad de Ciencias Económicas \\ Universidad Central "Marta Abreu" de Las Villas
}

\begin{abstract}
This work aims to develop a knowledge management model for companies in the goods retail sector in Villa Clara. An extensive literature on knowledge management was carried out and through an empirical analysis, the need to implement knowledge management in this kind of businesses is studied. Finally, a model constructed upon analytical instruments is proposed and validated by experts in the province of Villa Clara.
\end{abstract}

KEY WORDS

Trade, knowledge, management, retail, model. 


\section{Introducción}

El conocimiento organizacional ha tomado gran importancia internacional en las dos últimas décadas. En los países con menos desarrollo se ha despertado el interés por sus particularidades. La gestión del conocimiento es un proceso a través del cual se obtiene el conocimiento necesario para su internalización como recurso en la empresa. Los recursos humanos, su talento y sus capacidades son el centro de atención en busca del éxito en la gestión empresarial.

En algunos países de América Latina se aboga por la inclusión social en la educación y la salud, con el fin de crear el potencial humano requerido para la gestión del conocimiento. Las naciones con más alto desarrollo en Europa y Norteamérica utilizan la digitalización de la sociedad y los sistemas automatizados de gestión del conocimiento para la toma de decisiones y el aprendizaje continuo.

En el caso cubano, desde el año 2000 se han introducido conceptos y técnicas de gestión del conocimiento en algunas empresas, en su mayoría aquellas que ofrecen productos intangibles, entiéndase empresas biotecnológicas y centros de investigación que, por tanto, utilizan de manera intensiva el conocimiento como insumo. Las empresas de otros sectores de la economía han dado pasos exiguos en la incorporación de algunos principios de gestión del conocimiento, pero no existen resultados concretos que avalen una propuesta cubana en el tema.

Cuba posee características especiales para introducir la gestión del conocimiento en las empresas de cualquier sector y una cultura de economía basada en el conocimiento que lo propicia. Esto se debe a que la población tiene una formación técnica o profesional consolidada y se prioriza la educación y superación desde los niños hasta los adultos. Los servicios médicos, de educación y el turismo tienen un papel determinante en la economía cubana y son sectores con alto componente de conocimiento incluido. El sector del comercio es medular en el proceso de acercamiento de la producción industrial y agropecuaria al consumidor. El comercio ha tenido, desde la década de los noventa, cambios importantes que afectan la canasta básica de la familia cubana.

La gestión del conocimiento puede contribuir a la integración de todos los portadores de conocimiento útil para el comercio con el fin de cumplir su rol económico y social de acortar el ciclo productivo, la reproducción de la economía y la acumulación de riqueza social, teniendo en cuenta que la innovación 
y la creación de riquezas utilizan como insumo medular el conocimiento acumulado y la capacidad para incrementarlo y aprovecharlo. Por tanto, es menester de los economistas cubanos de estos tiempos adecuar los principios de la gestión del conocimiento a las particularidades de las empresas cubanas en el proceso de transición al socialismo en el que están inmersos, en el sector de los servicios y especialmente en el comercio.

Constituye el problema del presente trabajo el hecho de que desde 2012 se estableció en el VI Congreso del Partido Comunista de Cuba la política de lineamientos que determina el curso a seguir en el largo plazo para la actualización del modelo económico cubano. Se puede establecer una conexión concreta entre algunos aspectos tratados en esta política y la gestión del conocimiento, lo cual demuestra la relevancia y necesidad de esta disciplina en las empresas cubanas de comercio minorista de bienes para la nueva etapa de ajustes. Este sector posee un stock de conocimientos en sus recursos humanos, sus documentos y máquinas que no es utilizado de manera intensiva para hacer frente a la falta de correspondencia interna y entre sectores, lo cual ha incidido en la falta de eficiencia general del sector objeto de estudio. En este sentido, lo que el consumidor cubano demanda y lo que los sectores primario y secundario tienen capacidad para producir deben estar en correspondencia a través de la comunicación. Villa Clara cuenta con cinco empresas estatales y un grupo empresarial dedicado al comercio minorista de bienes, los cuales deben implementar estos principios para usar de manera sistemática el conocimiento útil que poseen.

El problema científico que se define es cómo contribuir a la implementación de la gestión del conocimiento en empresas de comercio minorista de bienes en Villa Clara a través de un modelo. Hay que considerar que este debe servir de guía conceptual y metodológica para implementar la gestión del conocimiento en este tipo de organizaciones. Entonces, el objetivo general de la investigación es elaborar un modelo de gestión del conocimiento para empresas pertenecientes al sector del comercio minorista de bienes en Villa Clara. En tanto, la hipótesis de trabajo es la siguiente: si se elabora un modelo de gestión del conocimiento para empresas de comercio minorista de bienes se contribuirá a elevar la eficiencia del sector en Villa Clara.

Se comienza con una explicación de los conceptos fundamentales: conocimiento organizacional, conocimiento tácito, conocimiento explícito y gestión 
del conocimiento. Después se analizan las etapas investigativas para la elaboración del modelo de gestión del conocimiento y, finalmente, se presenta el modelo en general y su validación por el método de expertos.

El presente trabajo puede ayudar a adecuar determinados conceptos y principios, elaborados en otros contextos, a la actualidad económica de la isla de Cuba. Las empresas cubanas de comercio deben considerar incorporar este modelo que involucra un conjunto de técnicas en pro de elevar el uso del conocimiento organizacional. El proceso de gestión empresarial y sus resultados económicos podrían mostrar un impacto favorable por esta incorporación.

\section{La conformación del conocimiento organizacional}

La doctora Gloria Ponjuán Dante define el conocimiento organizacional como:

el conjunto de cogniciones y habilidades con las cuales los individuos suelen solucionar problemas, comprenden la teoría, la práctica, las reglas cotidianas y las instrucciones para la acción. El conocimiento se basa en datos e información, pero a diferencia de estos, siempre está ligado a las personas. Forma parte integral de los individuos y representa las creencias de estos acerca de las relaciones causales [...] Solo es conocimiento cuando alguien puede identificar y comprender los patrones y sus implicaciones [2003: 58].

Por tanto, el conocimiento es un recurso indispensable para la toma de decisiones y para el aprendizaje que propicia el mejoramiento en el desempeño empresarial. Ponjuán Dante presenta una de las características principales del conocimiento: si se concibe como factor productivo, por su apego a las personas, es una cualidad intrínseca de la fuerza de trabajo, la cual es portadora y creadora de conocimiento durante el proceso de creación de riquezas. El conocimiento debe seguir determinados patrones de tangibilización y comprensión para poder ser transferido y compartido con otros y tenido en cuenta como recurso productivo. Pero no basta con los datos y la información como base, deben considerarse las capacidades naturales o adquiridas por las personas que les permiten utilizar el conocimiento organizacional. 


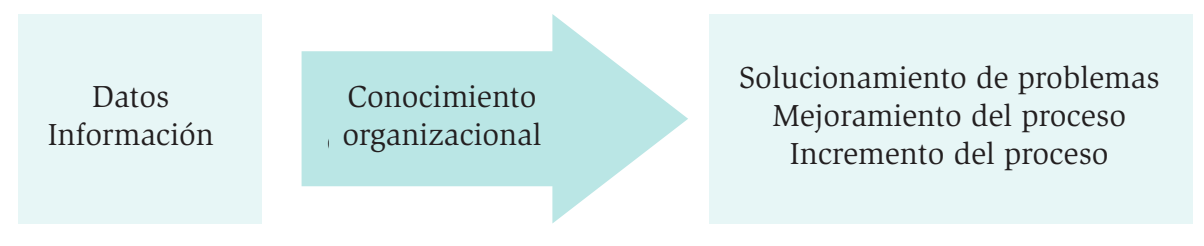

Fuente: Elaboración propia

Figura 1. Conformación del conocimiento organizacional

Esta concepción sobre el conocimiento organizacional es reforzada por el autor español Manuel Riesco González, cuando plantea que "el conocimiento en las empresas es un conjunto abierto de ideas, creencias y experiencias contrastadas, y asimiladas mediante la reflexión que sirve de guía para la acción. Se encuentra incrustado en los individuos, los grupos, las organizaciones y el entorno social" (2006: 66). Es decir, el conocimiento organizacional es el conjunto de todos los recursos intangibles con que cuenta una institución, que pueden ser utilizados para alcanzar los objetivos propuestos y que tienen como soporte y creadoras fundamentales a las personas.

Además, "el conocimiento es reconocido como un producto social, por cuanto se construye socialmente como un producto de la actividad de los hombres, en donde cada individuo adquiere gran parte de su saber a partir de su propia experiencia, pero considerando una asociación con los demás y un aprendizaje inteligente desde esa cotidianidad. Este saber es acumulado por la sociedad y distribuido, en forma explícita e implícita” (Ollarves Levison, Estévez González y Salguero, 2012: 20). El conocimiento tiene su base también en las estructuras sociales y las políticas que se establecen para la creación de conocimiento en las organizaciones. Por ende, se puede señalar que el conocimiento organizacional es el conjunto de recursos intangibles que posee la organización que tienen relevancia social, están soportados en las personas y los documentos y son significativos en el logro de los objetivos empresariales.

El conocimiento organizacional puede clasificarse de diversos modos de acuerdo con la perspectiva con que se estudie. Una de las clasificaciones más utilizadas en la literatura científica sobre el tema es la del conocimiento tácito y el explícito, que abarca todo el contenido del objeto de estudio porque tiene en 
cuenta su naturaleza, su origen y su soporte, así como la forma en que se manifiesta, y entiende el conocimiento como un recurso del proceso productivo.

El conocimiento tácito es el que no ha sido estructurado de manera comprensible para otras personas. Desde el punto de vista de la ciencia, es "el empírico, implícito, tácito o sensitivo, que de manera no sistemática da cuenta de lo aparencial y, en mayor o menor medida, de cómo este oculta ciertos elementos esenciales” (Triana Cordoví, Torres Pérez y Martín Fernández, 2005: 55). Esta definición no brinda el soporte fundamental de este conocimiento, que es la mente de las personas, pero explica su relación con la manera en que se percibe un problema y se le da solución en la práctica individual. Es una percepción personal de los fenómenos que ha sido asumida por la experiencia vivida.

Conocimiento

organizacional

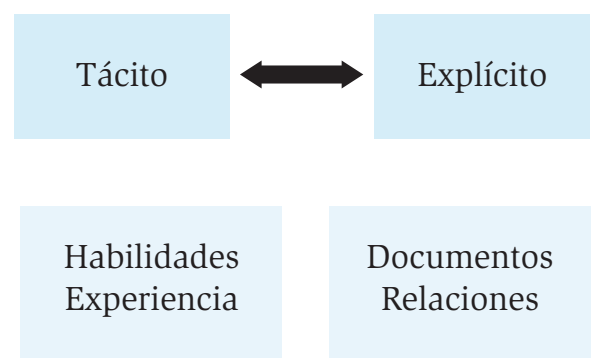

Fuente: Elaboración propia.

Figura 2. Tipos de conocimiento y formas en que se manifiestan 
El conocimiento explícito, por otro lado, es el "conocimiento que puede ser transferido de un individuo a otro usando algún sistema de símbolos (...) Es un conocimiento que ha sido codificado de alguna manera y puede comunicarse 0 difundirse con facilidad” (Ponjuán Dante, 2006: 13). Este tipo de conocimiento viabiliza la permanencia de tal recurso en la empresa y su incorporación a la producción por personas ajenas a su creador en forma de documentos o relaciones.

Fidel Castro Díaz-Balart propone que "el conocimiento formal o explícito es el que se puede transmitir mediante signos escritos. Las fuentes de este tipo de conocimiento son muy variadas, por ejemplo: revistas técnicas, catálogos comerciales, libros, patentes, planos técnicos, diseños o tesis doctorales” (2002: 300). Es importante ejemplificar las fuentes de conocimiento explícito. En todo documento donde se plasme el resultado de una experiencia y el quehacer organizacional en forma estructurada, y que sea útil para la actividad económica en cuestión, habrá conocimiento explícito.

En resumen, el conocimiento tácito se encuentra en la mente de las personas y es inseparable de ellas, no ha sido codificado o estructurado. Por otra parte, el conocimiento explícito es aquel que se ha llevado a códigos o estructuras que permiten su transferencia y es permanente en la empresa, porque se queda en ella cuando el creador se marcha.

\section{Gestión del conocimiento en la empresa}

La gestión del conocimiento comprende pasos lógicos a seguir, se considera un proceso y se puede descomponer en funciones diversas centradas en el conocimiento de las personas de la empresa. Por otro lado, el proceso de gestión del conocimiento no puede desligarse del proceso productivo. El esfuerzo debe dirigirse al logro de objetivos específicos enfocados en la eficiencia y la eficacia empresariales. Esta idea es reforzada por el investigador cubano Eduardo Orozco Silva y por Juan R. Carro Suárez, quienes entienden que la gestión del conocimiento "reconoce y utiliza el valor más importante de las organizaciones: el recurso humano y el conocimiento que los humanos poseen y aportan a la organización” (2002: 18). Reconocer un conocimiento ya permite su utilización como recurso en un contexto y con una finalidad determinada. Las personas son el centro de su atención y la explicitación de su conocimiento lo es de la gestión del conocimiento. 
Desde el punto de vista de la administración, el criterio del investigador español Víctor Jesús García Morales es que la gestión del conocimiento "es la función que planifica, coordina y controla los flujos de conocimientos, estando formada por varios procesos interrelacionados tales como el proceso de adquisición de conocimiento, procesos de creación de conocimiento y proceso de internalización de conocimiento" (2005: 65). En correspondencia con este planteamiento, se puede decir que el proceso de gestión del conocimiento es una función que implica la toma de decisiones de los directivos y debe seguir las etapas del ciclo administrativo conocidas como planificación, organización, dirección y control. Estas se manifiestan en los diferentes subprocesos realizados como parte de la gestión, que son la adquisición, creación e internalización del conocimiento, según García Morales.

En las empresas, el conocimiento se gestiona con una finalidad esencialmente económica. No es conveniente dilapidar esfuerzos en función del conocimiento si no se ha demostrado que tendrá un rendimiento económico para la organización. "El principal recurso con que cuentan las organizaciones hoy en día es el conocimiento; sus costos son elevados pero genera un importante retorno sobre la inversión. La productividad del conocimiento será cada vez más decisiva en el éxito económico de un país o una empresa" (Pérez Frías y Pérez R., 2004: 346). Así pues, toda inversión o esfuerzo que se realice para la gestión del conocimiento debe hacerse pensando en los rendimientos que tendrá en un plazo determinado, con la inherente autorrealización de las personas que producen (figura 3).

Se conoce, además, que:

la gestión o gerencia del conocimiento es la estrategia que utilizan las organizaciones para lograr que el conocimiento permanezca en la organización y no en personas, es decir, que el saber hacer de la organización no recaiga en cabeza de personas sino de procesos, permitiendo que el capital intelectual de la organización aumente, logrando generar ventajas competitivas sostenibles en el tiempo, y evitando que las personas sean indispensables, logrando minimizar sustancialmente el impacto negativo que se genera cuando el empleado no asiste a la organización por cualquier motivo [Saldarriaga Ríos, Alzate Molina y Gallego Álvarez, 2012]. 


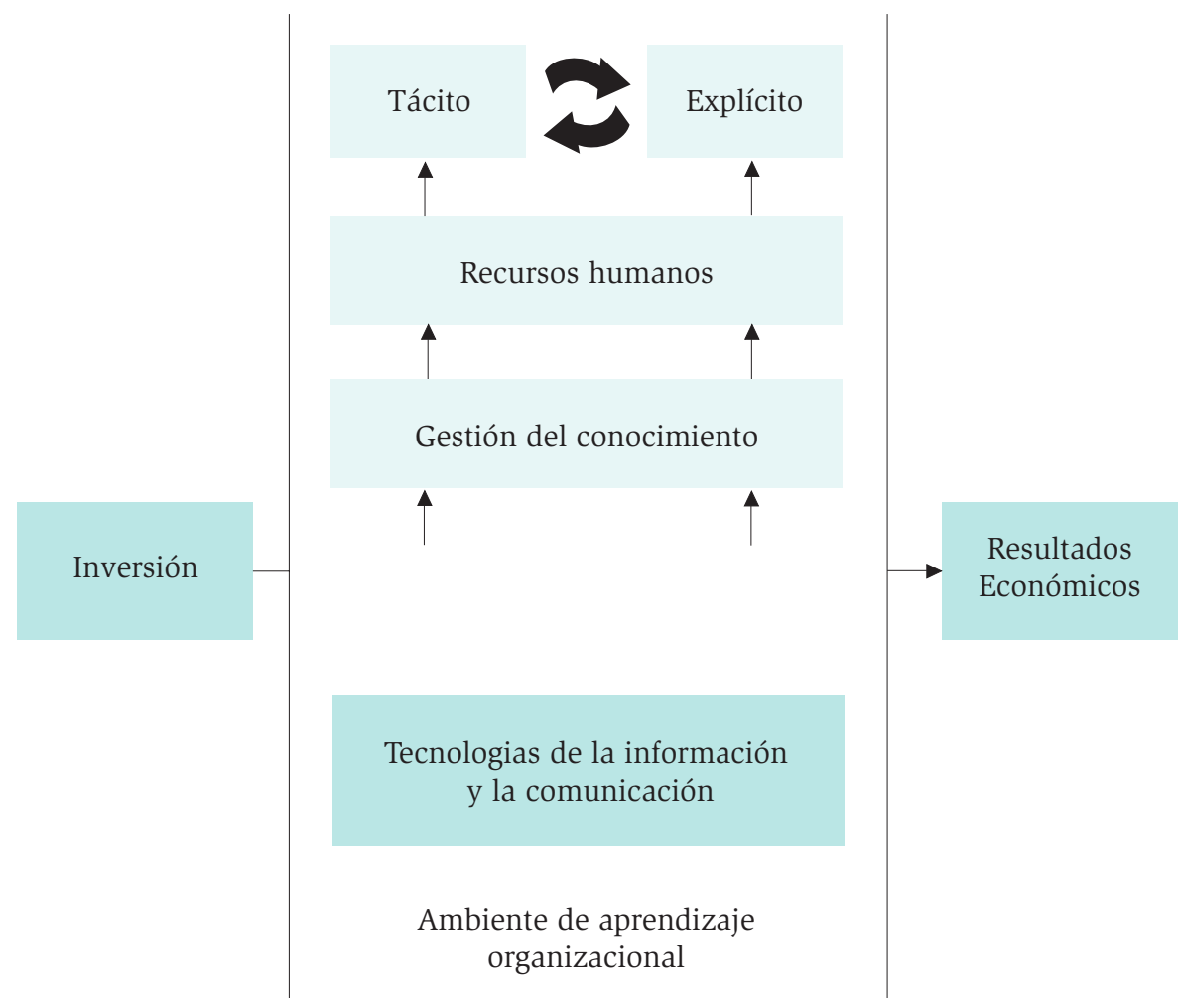

Fuente: Elaboración propia.

Figura 3. La gestión del conocimiento en la empresa

No se refiere solo a que el empleado pueda faltar, sino que también pueda irse de la empresa o fallecer. Por ello, la gestión del conocimiento debe trabajar sistemáticamente por explicitar todo el conocimiento en los documentos o en la cultura de la organización. En fin, la gestión del conocimiento es el proceso técnico y directivo que asegura la coordinación de los recursos intangibles de la organización y los pone a disposición de los objetivos de la institución. 


\section{Necesidad del conocimiento en el comercio minorista de bienes}

El comercio es uno de los sectores más importantes en cualquier economía. A través de este sector se acorta el ciclo productivo industrial y se contribuye a la reproducción del mismo. Se puede clasificar en mayorista o minorista. El doctor Roberto Pons García, Zhao Hui Yuan y Dayana Duffus Miranda plantean que "tiene función de aproximar, repartir, distribuir. Todas las personas dedicadas a llevar a cabo esta actividad son denominadas detallistas, minoristas y los comerciantes, etc. El lugar donde se lleva a cabo dicha actividad también tiene varias denominaciones como son comercio, tienda, almacenes, y lo ya denominado punto de venta" (2008). En este concepto destacan las denominaciones del personal que se dedica al comercio, el cual debe tener una formación y capacidades adecuadas al sector, por las constantes relaciones que se establecen. Es decir, algunos de los procesos más rigurosos en la gestión del conocimiento en el comercio minorista de bienes son la selección y la formación del personal. También el ambiente del punto de venta merece especial atención según las exigencias del consumidor, y el personal debe tener habilidades para esta actividad.

Según Carlos Marx, el ciclo del capital tiene tres fases. La primera es la compra de las mercancías que constituyen los insumos del proceso productivo, esencialmente fuerza de trabajo y medios de producción (D-M). La segunda es la producción como tal, la transformación de las mercancías en mercancías de un valor superior (P). La tercera es la venta de las mercancías producidas, la comercialización de la producción (M'-D'). Se dice que M' y D' son M y D incrementados por la plusvalía (Marx, 1973: 27). Además, Marx explica que lo que se invirtió inicialmente en mercancías regresa convertido en dinero y asegura la reproducción del sector industrial. Este sector no se autoabastece, sino que depende de manera relativa de la eficiencia de las empresas productoras de bienes para realizar su actividad vital: la comercialización. Por ser un sector de servicios, depende en mayor medida de la capacidad de esa fuerza de trabajo y de sus habilidades, así como de la competitividad de sus suministradores.

En la figura 4 se muestra la clasificación del comercio minorista de bienes en Cuba. El comercio estatal incluye a las principales empresas de propiedad estatal; puede ser normado a través de la libreta de racionamiento, liberado en peso convertible cubano (cuc) (entiéndase las tiendas de recaudación de divisas creadas desde los años noventa como medida para la recuperación de la crisis 
producida después de la caída del campo socialista y de la uRss) y liberado en moneda nacional (CUP); se refiere también a las tiendas de los grupos empresariales de comercio que venden en el peso tradicional. El comercio no estatal puede ser cooperativo o privado, y el ilegal genera un efecto negativo en el marco de las empresas estatales, desde donde se desvían recursos, y en toda la economía por el desplazamiento de las ventas del sector legal.

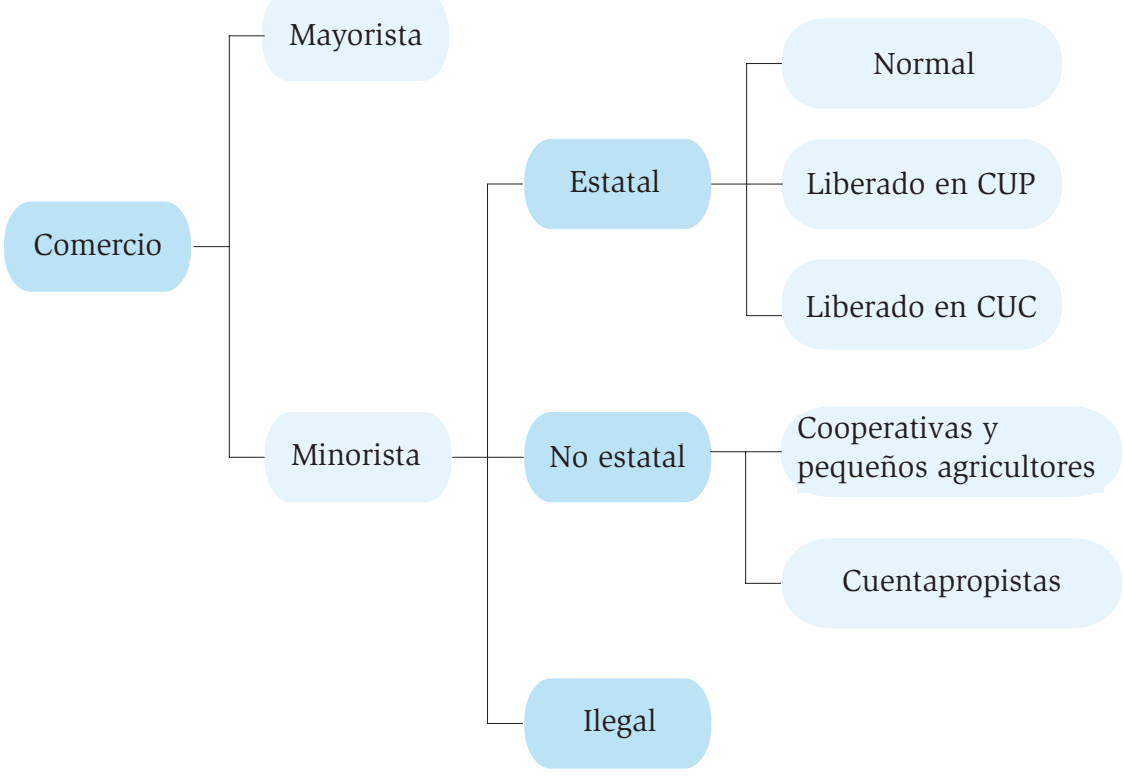

Fuente: Elaboración propia

Figura 4. Estructura del sector del comercio minorista de bienes en Cuba

Este sector requiere investigaciones que contribuyan a elevar su eficiencia general y a aprovechar sus recursos creados para este fin. Hasta el momento no se ha modelado la gestión del conocimiento en estas empresas, por lo que se han seguido los pasos que se explican a continuación.

Fase 1. Determinar los indicadores de eficiencia general del sector. Para ello se ha consultado a los directivos de las empresas del sector en la provincia de Villa 
Clara. Estas empresas tienen como línea de negocio la de comercio minorista de bienes o se dedican solo a ella. Las empresas consultadas son:

- Sucursal Artex de Villa Clara

- Sucursal Caracol Villa Clara

- Cadena de tiendas TRD Caribe (División Centro)

- Corporación Cimex Villa Clara

- Fondo Cubano de Bienes Culturales (Villa Clara)

- Grupo Empresarial de Comercio de Villa Clara

Fase 2. Definir los procesos clave de la empresa que proporcionan satisfacción al cliente. Se realizó una entrevista a clientes especializados de las empresas de comercio minorista de bienes. Los clientes son toda la población que consume los bienes que se requieren para la vida. Al seleccionar a los clientes especializados se consideraron cualidades como:

- Mayoría de edad

- Cliente frecuente de los puntos de venta en Villa Clara

- Formación que permita dar criterios especializados

- Vínculo laboral ajeno al sector, para no parcializarse en los criterios

Fase 3. Detectar los conocimientos que influyen directamente en los procesos clave de la empresa. Se consultó a trabajadores y especialistas de empresas de comercio minorista de bienes. Se trabajó con personal de las empresas mencionadas en la fase 1. Los trabajadores y especialistas se seleccionaron de diferentes áreas y edades, con experiencia en el sector y con formación adecuada para dar un criterio especializado.

Fase 4. Validar el modelo propuesto para la gestión del conocimiento en el comercio minorista de bienes. Primero se recurrió a expertos que evaluaron el modelo en cuanto a los siguientes criterios:

- Carácter integrador: es posible integrarlo al conjunto de procesos que forman parte de las empresas de comercio minorista de bienes como una concepción completa de los recursos de conocimiento en tal contexto. Incluye todos los elementos básicos de la gestión del conocimiento en este tipo de empresas. 
- Valor teórico: aclara los marcos conceptuales en los que debe basarse la implementación de la gestión del conocimiento en empresas de comercio minorista de bienes según sus características específicas. Es exhaustivo en cuanto a las categorías que deben considerarse y delimita su alcance y su interconexión con claridad. Puede utilizarse como base para la capacitación inicial del personal que estará implicado en su aplicación.

- Valor metodológico: constituye una guía para la comprensión de relaciones entre los conocimientos con que cuentan estas empresas, los procesos internos en los que estos influyen y los resultados esperados a nivel de la empresa con la aplicación. Está articulado de forma que puede implementarse a través de un procedimiento lógico.

- Valor práctico: se puede comprobar su viabilidad en la práctica de las empresas de comercio minorista, su utilización en la realidad como guía teórico-metodológica y la factibilidad de su aplicación desde el punto de vista económico y social. Es fácil de entender.

Después se calcularon las medidas de tendencia central y el índice de consenso de expertos (ISC) para que el modelo quedara validado.

\section{La eficiencia en el comercio minorista de bienes}

El modelo de gestión del conocimiento debe contener los indicadores de medición de su efecto en la empresa. Para determinar estos indicadores se aplicó una entrevista a directivos que, por su desempeño, están familiarizados con el manejo del término eficiencia. La muestra que se tomó fue intencional y se tuvieron en cuenta como criterios de intención las diferentes características que avalan su experiencia en el sector y sus conocimientos:

1. Empresas en las que han trabajado

2. Cargos que han ocupado

3. Años de experiencia en el sector objeto de estudio

4. Formación técnica y académica

Los entrevistados fueron ocho directivos de las principales empresas de la provincia de Villa Clara, en la región central de Cuba; gerentes de empresas y 
de tiendas, personal con más de diez años de experiencia y con una formación académica en general de licenciados o máster.

Las preguntas abiertas que se aplicaron a la muestra fueron:

•¿Qué elementos determinan su satisfacción en el punto de venta?

• ¿En qué medida influye cada uno de estos elementos en que usted se sienta satisfecho?

\section{Resultados}

- En las instituciones estudiadas, la eficiencia es tratada como el correcto logro de los resultados de la gestión empresarial, vistos como la utilidad obtenida en cada periodo.

- No basta con obtener una utilidad, esta debe complementarse con un aumento de la calidad del servicio, la superación y el buen desempeño del personal, puntos de ventas estratégicos que apoyen la imagen de la empresa, cumplimiento de las tareas, productos de calidad, un precio que corresponda con la misma, y una dirección que sepa guiar a las instituciones hacia las metas trazadas.

- Es de vital importancia que en las organizaciones se trabaje con eficacia; en todas ellas es necesario cumplir con las metas trazadas y con los objetivos que se proponen con el uso óptimo de los recursos.

- La utilidad está en el centro de todo el proceso pero se debe complementar con los factores mencionados, aunque estos parezcan más subjetivos, para abarcar todo el desempeño de la organización.

- La eficiencia con que se trabaje en las empresas influye en la satisfacción del cliente, el cual tiene el papel principal, pues sin él estas empresas no tienen razón de ser, y la única manera de aumentar las utilidades y sobrevivir en las condiciones del entorno económico existente es que estas sean capaces de satisfacer sus necesidades.

- La información es un elemento de gran valor dentro de cualquier organización; tiene que renovarse constantemente para que las organizaciones sean capaces de enfrentar los cambios bruscos del entorno turbulento que 
caracteriza a la economía del momento; la eficiencia en las empresas propicia que esta información sea confiable, que se encuentre en el momento justo para la toma de decisiones y que se tenga una imagen global de toda la organización, visión muy necesaria para su correcto desempeño.

En resumen, los indicadores fundamentales y más integradores de eficiencia en el sector del comercio minorista de bienes son el incremento de las utilidades y la satisfacción del cliente, los cuales se desarrollan a continuación.

- Incremento de las utilidades. Se define como la parte del monto de las utilidades que se obtiene por la implementación de la gestión del conocimiento en la empresa, es decir, por el aprovechamiento del conocimiento organizacional. Incluye la determinación de los costos de implementación en partidas adecuadas, calculando todos los gastos de materiales, horas de trabajo de empleados y otros egresos en que pueda incurrirse por la aplicación. Se deben estimar los ingresos por utilización del conocimiento, esto es, el aumento de los ingresos por la aplicación de las técnicas de aprovechamiento del conocimiento en la empresa, lo cual puede hacerse con la ayuda de los especialistas en economía y en comercialización en conjunto. Finalmente se calcula la ganancia o pérdida, resultado que se obtiene como valor agregado por la aplicación de las técnicas de aprovechamiento del conocimiento.

- Satisfacción del cliente. Es el estado de complacencia del cliente con el servicio que recibe en los puntos de venta, lo cual incluye la correspondencia entre los bienes que consume en la entidad y su necesidad real, la atención que recibe por parte del personal en contacto y el ambiente del punto de venta. Se puede determinar mediante clientes especializados que evalúen la mejora en el servicio por la implementación de técnicas de gestión del conocimiento. Ellos deben ser capaces de expresar su satisfacción antes y después de la aplicación de la gestión del conocimiento para visualizar las mejoras en cuanto a la gestión de la oferta, la atención al cliente y el diseño del punto de venta. 


\section{Procesos clave del comercio minorista de bienes}

Una vez determinados los principales indicadores de eficiencia en el comercio minorista de bienes, se aplicó otra entrevista semiestructurada a clientes especializados del sector para evaluar la importancia de los diferentes procesos clave y el porcentaje en que influyen en la satisfacción del cliente. En total fueron 16 clientes especializados que frecuentan sistemáticamente los puntos de venta de todas las empresas. Todos estaban entre los 20 y los 70 años de edad. De ellos, 11 son mujeres y cinco hombres. Todos tenían nivel escolar técnico o profesional y formación relacionada con la contabilidad, la economía o el comercio, y habían desempeñado o desempeñan responsabilidades afines a su formación.

Los procesos evaluados por los clientes especializados fueron la gestión de la oferta, la atención al cliente y el diseño del punto de venta. Se les cuestionó sobre el nivel de importancia de cada proceso y el porcentaje de satisfacción que le proporcionaba cada uno (cuadros 1 y 2).

Cuadro 1. Procesos clave del comercio minorista de bienes

\begin{tabular}{|c|c|}
\hline Proceso & DESCRIPCIÓN \\
\hline Gestión de la oferta & $\begin{array}{l}\text { Es el proceso que asegura que los productos exhibidos en el } \\
\text { punto de venta correspondan con lo que el cliente necesita. Co- } \\
\text { mienza con la determinación de necesidades concretas en los } \\
\text { consumidores, después se evalúan los proveedores disponibles } \\
\text { en cuanto a precio, calidad requerida, variedad, imagen del pro- } \\
\text { ducto, durabilidad y funcionalidad; también se tienen en cuenta } \\
\text { las posibilidades materiales y financieras de la empresa para } \\
\text { asumir la compra. Finalmente se transportan los bienes hasta el } \\
\text { punto de venta en el momento adecuado. }\end{array}$ \\
\hline Atención al cliente & $\begin{array}{l}\text { Es el nivel de comunicación y asesoramiento que recibe el clien- } \\
\text { te en el punto de venta por el personal en contacto. Tiene que } \\
\text { ver con el nivel de empatía que el personal mantiene con el } \\
\text { cliente en el momento del servicio, la información que ofrece } \\
\text { sobre las cualidades del producto y la orientación o imagen que } \\
\text { proyecta sobre el punto de venta y la empresa. Este proceso em- } \\
\text { pieza con el reclutamiento y selección del personal que prestará } \\
\text { el servicio en el punto de venta. Se le debe evaluar sistemáti- } \\
\text { camente en función de lo que el cliente espera de la empresa } \\
\text { en términos de atención y se le debe capacitar para mejorar sus } \\
\text { desviaciones. }\end{array}$ \\
\hline
\end{tabular}


Cuadro 1. Procesos clave del comercio minorista de bienes

(finaliza)

Diseño de los puntos de venta

Cuadro 2. Análisis estadístico del nivel de importancia de los procesos

\begin{tabular}{|c|c|c|c|}
\hline EstAdÍGRAFOS /PROCESO & GESTIÓN DE LA OFERTA & ATENCIÓN AL CLIENTE & $\begin{array}{c}\text { DisEÑO DE LOS PUNTOS } \\
\text { DE VENTA }\end{array}$ \\
\hline Mediana & 5 & 5 & 5 \\
\hline Moda & 5 & 5 & 5 \\
\hline Media & 4.6875 & 4.625 & 4.3125 \\
\hline Desviación estándar & 0.47871355 & 0.71879529 & 0.94648472 \\
\hline ISC & $99 \%$ & $98.5 \%$ & $97.6 \%$ \\
\hline
\end{tabular}

Fuente: Elaboraciòn propia.

Por ser mayor que $85 \%$ en todos los casos, los tres procesos quedan aprobados por los clientes como clave para la eficiencia en el sector del comercio minorista de bienes (gráfica 1).

Si se analiza de forma tendencial, es decir, usando la media aritmética, en la gráfica 1 se aprecia el nivel de importancia que los clientes otorgan a cada proceso. La gestión de la oferta es el más relevante, seguido por la atención al cliente y, al último, el diseño del punto de venta.

La satisfacción del cliente depende de estos procesos en cierto porcentaje, que quedó establecido con esta técnica. La gestión de la oferta determina 50 \% de la satisfacción del cliente, la atención que recibe por parte del personal lo hace en $30 \%$, y el ambiente o diseño del punto de venta, en $20 \%$ (gráfica 2). 


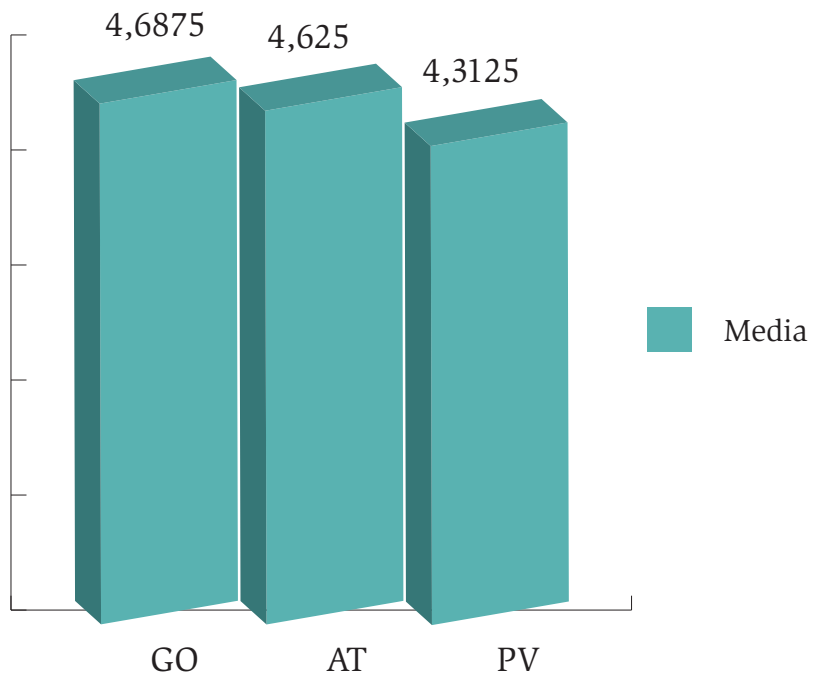

Go (gestión de la oferta) AT (atención al cliente) Pv (diseño del punto de venta) Fuente: Elaboración propia.

Gráfica 1. Importancia tendencial de los procesos clave

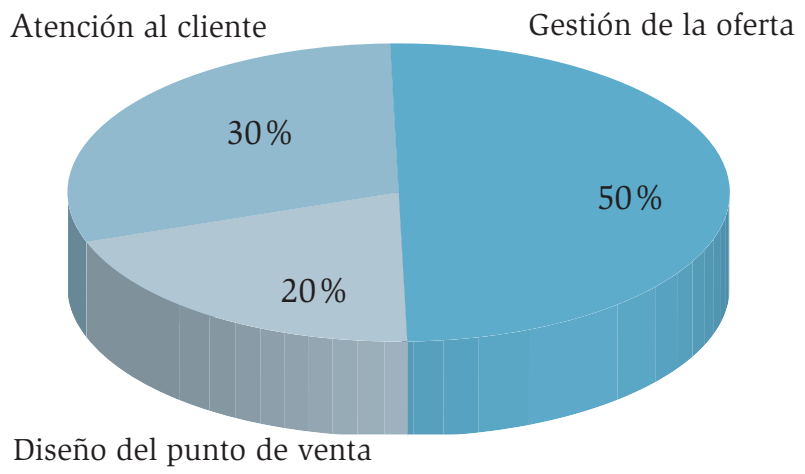

Fuente: Elaboración propia. 
Se puede concluir que los tres procesos tienen una determinada importancia para la eficiencia en el sector del comercio minorista de bienes según la perspectiva del cliente, y se está de acuerdo en que cada uno aporta una parte de su satisfacción con el servicio. Esta técnica ayuda a comprender la prioridad de los clientes en cuanto al servicio.

\section{Conocimiento organizacional en las empresas de comercio minorista}

Luego de establecer los principales indicadores de eficiencia, su importancia y el porcentaje en que los procesos clave satisfacen al cliente, se aplicó una encuesta a trabajadores de las diferentes instituciones con el objetivo de evaluar la utilización de los conocimientos y el nivel de importancia que se le otorga en el desempeño de la empresa.

Las formas en que se manifiesta el conocimiento en el comercio minorista de bienes y su descripción se determinaron a partir de una revisión bibliográfica de otros modelos de gestión del conocimiento que expresan las formas concretas en que se visualiza el conocimiento organizacional. Los modelos que más aportaron a la revisión fueron:

- Cuadro de mando integral (Kaplan y Norton, 1996)

- Navigator de Skandia (Edvinsson, 1992-1996)

- Universidad de West Notario (Bontis, 1996)

- Technology Broker (Brooking, 1996)

- Knowledge Management Assessment Tool (Kmat, 1999)

- Modelo Nova, Club de Gestión del Conocimiento de la Comunidad Valenciana (Camisón, Palacios y Devece, 1999)

- Modelo de dirección estratégica por competencias: el capital intangible (Bueno, 1998)

- Modelo de gestión del conocimiento de KPмG Consulting (Tejedor y Aguirre, 1998)

- Modelo Intelect (Euroforum, 1998)

Con los resultados fundamentales de la revisión se realizó una lluvia de ideas con seis especialistas de la Universidad Central "Marta Abreu" de Las 
Villas, todos ellos doctores o másteres y con más de cinco años como investigadores en el tema del comercio en Cuba.

Cuadro 3. Formas en las que se manifiesta el conocimiento organizacional

\begin{tabular}{|ll|}
\hline \multicolumn{1}{|c}{ Conocimiento } & \multicolumn{1}{c|}{ Descripción } \\
\hline Habilidad & $\begin{array}{l}\text { Demostración de una capacidad a través de la realización de } \\
\text { una acción. }\end{array}$ \\
\hline Formación & $\begin{array}{l}\text { Títulos de enseñanza media, técnicos o superiores que poseen } \\
\text { los empleados en correspondencia con el cargo o responsabili- } \\
\text { dad que ocupan. }\end{array}$ \\
\hline Capacitación & $\begin{array}{l}\text { Actividades preparatorias, continuas y planificadas en la } \\
\text { organización con el fin de mejorar el desempeño. }\end{array}$ \\
\hline Experticia & $\begin{array}{l}\text { Conocimiento que poseen los especialistas o el personal de } \\
\text { más experiencia en la organización. }\end{array}$ \\
\hline Manuales & $\begin{array}{l}\text { Documentos que sirven de guía para la acción de la empresa: } \\
\text { procedimientos, normas, metodologías o manuales. }\end{array}$ \\
\hline Estructuras & $\begin{array}{l}\text { Relaciones formales e informales que se establecen entre las } \\
\text { personas y los medios que aseguran el desarrollo de los } \\
\text { procesos en la organización. }\end{array}$ \\
\hline Relación con el entorno & $\begin{array}{l}\text { Nivel de conexión y conocimiento sobre los agentes externos } \\
\text { que influyen en los resultados de la empresa (clientes, provee- } \\
\text { dores, banco y otros). }\end{array}$ \\
\hline Imagen e identidad \\
Estilo y conducta percibidos por los agentes externos de la \\
empresa que le permiten diferenciarla del resto.
\end{tabular}

Fuente: Elaboraciòn propia.

Los trabajadores del sector que se seleccionaron para aplicar la técnica fueron personas con experiencia en el ramo, con un nivel técnico o académico apropiado. Fueron un total de 18 trabajadores de las diferentes empresas de la provincia de Villa Clara, todos con más de diez años de experiencia en el sector, con nivel técnico o superior, y con puestos de directivos, vendedores, almaceneros, administradores o jefes de calidad. 
Cuadro 4. Importancia de cada tipo de conocimiento (\% de trabajadores)

\begin{tabular}{|c|c|c|c|c|c|c|}
\hline Núm. & Conocimiento/importancia & $\begin{array}{l}\text { Muy } \\
\text { baja }\end{array}$ & Baja & Mediana & Alta & $\begin{array}{r}\text { Muy } \\
\text { alta }\end{array}$ \\
\hline 1 & Habilidades & & 5.6 & 5.6 & 50.0 & 38.9 \\
\hline 2 & Formación & & 5.6 & 16.7 & 22.2 & 55.6 \\
\hline 3 & Capacitación & & 5.6 & 11.1 & 27.8 & 55.6 \\
\hline 4 & Experticia & & & 11.1 & 55.6 & 33.3 \\
\hline 5 & Manuales & & 5.6 & 5.6 & 5.6 & 83.3 \\
\hline 6 & Estructuras & 5.6 & & 16.7 & 55.6 & 22.2 \\
\hline 7 & Relación con el entorno & & 5.6 & 5.6 & 38.9 & 50.0 \\
\hline 8 & $\begin{array}{l}\text { Imagen e identidad } \\
\text { corporativas }\end{array}$ & & & 5.6 & 27.8 & 66.7 \\
\hline
\end{tabular}

Fuente: Elaboraciòn propia.

\section{Resultados:}

- El conocimiento menos favorecido en importancia son las estructuras, porque este sector no crea bienes sino que agrega valor a partir del servicio de venta; la actividad fundamental, es decir, la venta, se desarrolla con estructuras muy simples en el punto de venta, aunque aquí se puede ignorar el papel que juega la gestión de la oferta, que es un proceso mucho más complejo.

- La formación y capacitación se consideran menos relevantes, teniendo en cuenta que estas no siempre se relacionan directamente con la mejora de la calidad del servicio y de las habilidades del personal, aunque pueden estar subestimadas por la pericia con que se labora en estas empresas, que obstaculiza los procesos de formación y capacitación.

- El conocimiento más destacado es la identidad e imagen corporativas, con $94.5 \%$ de trabajadores que las consideraron con una importancia alta 0 muy alta. Este conocimiento es el más abarcador pues reúne toda la cultura organizacional que se posee y se proyecta al entorno para alcanzar los objetivos de la empresa. 
- Otros tipos de conocimiento, como las habilidades, los manuales y la experticia, pueden constituir el fundamento de un servicio de calidad; los manuales como el referente básico de la acción organizacional, y las habilidades y la experticia como su reflejo más concreto en los empleados.

La mayoría de los trabajadores le asignaron una importancia alta o muy alta a cada conocimiento, por lo que se puede resumir que todos son parte del modelo y deben considerarse al implementar la gestión del conocimiento en este tipo de empresas. A partir de los resultados anteriores se calcularon medidas de tendencia central y el Isc.

Cuadro 5. Análisis estadístico del nivel de importancia de los conocimientos

\begin{tabular}{llllllllll}
$\begin{array}{l}\text { Estadígrafos/ } \\
\text { conocimientos }\end{array}$ & 1 & 2 & 3 & 4 & 5 & 6 & 7 & 8 \\
Moda & 5 & 5 & 5 & 4 & 5 & 4 & 5 & 5 \\
Media & 4.35 & 4.24 & 4.29 & 4.24 & 4.68 & 3.88 & 4.29 & 4.56 \\
Mediana & 5 & 5 & 5 & 4 & 5 & 4 & 4 & 5 \\
Desviación estándar & 0.786 & 0.970 & 0.919 & 0.664 & 0.862 & 0.992 & 0.849 & 0.618 \\
ISC & $92.1 \%$ & $90.2 \%$ & $91 \%$ & $93.3 \%$ & $91.3 \%$ & $90 \%$ & $91.5 \%$ & $93.8 \%$ \\
\hline
\end{tabular}

Fuente: Elaboraciòn propia.

En todos los conocimientos, el Isc supera $85 \%$, lo que permite constatar que los conocimientos evaluados tienen una gran influencia en el desempeño de las empresas del comercio minorista.

\section{Discusión de los resultados del modelo de gestión del conocimiento}

El modelo queda construido en tres partes. Los conocimientos de la empresa se pueden clasificar en tácitos o explícitos. Los conocimientos tácitos que deben tenerse en cuenta son: habilidades, capacitación, formación y experticia. Para estos, por su naturaleza, deben existir mecanismos de medición sistemática de su aprovechamiento y realizarse esfuerzos con el fin de volverlos explícitos. 
Los conocimientos explícitos en el comercio minorista de bienes son: estructuras, relaciones con el entorno, manuales, e imagen e identidad corporativas. En este caso, deben servir de base para el aprendizaje y la obtención de más conocimiento tácito, además deben incorporarse a la cultura organizacional.

Estos conocimientos tienen una influencia directa en los procesos clave de la empresa, los cuales son distintivos del sector. Estos procesos son: gestión de la oferta, que influye en 50 \% en la satisfacción del cliente; atención al cliente, con $30 \%$ de aporte a la satisfacción, y diseño del punto de venta, que contribuye en $20 \%$.

Sistemáticamente se deben medir los indicadores de eficiencia en los que se refleja el impacto de los principales procesos del comercio minorista de bienes, para visualizar el resultado de los esfuerzos que se dedican al conocimiento. Asimismo, se retroalimenta cada resultado a la gestión de los conocimientos con propuestas de mejora.

De manera integradora, el modelo debe funcionar como una guía de análisis de los esfuerzos de gestión del conocimiento. El punto de partida debe ser la potenciación de los conocimientos, su uso sistemático y su aprovechamiento intensivo. Cada forma en que se manifiesta el conocimiento debe ser gestionada en particular, enfocada al mejoramiento de los procesos clave determinados, fijando una relación causa-efecto entre cada conocimiento y proceso clave. El resultado final debe establecerse a través del incremento de la eficiencia con los indicadores detectados (figura 5).

Este modelo fue validado en su conjunto por expertos de las empresas del sector y de la academia en la región. Entre ellos hay doctores en Ciencias y másteres en Administración de Negocios graduados de la Universidad Central "Marta Abreu" de Las Villas, gerentes de empresas y especialistas con más de 20 años de experiencia en el sector (cuadro 6).

El modelo tiene un carácter integrador reconocido por los expertos, lo que afianza su trascendencia en el proceso de toma de decisiones en la implementación de la gestión del conocimiento en empresas de comercio minorista de bienes. Los valores teórico, metodológico y práctico se manifiestan en la necesidad de adecuación al sector, la lógica de su estructura y la utilidad en las decisiones operativas en la gestión del conocimiento y la gestión en general de la empresa. 


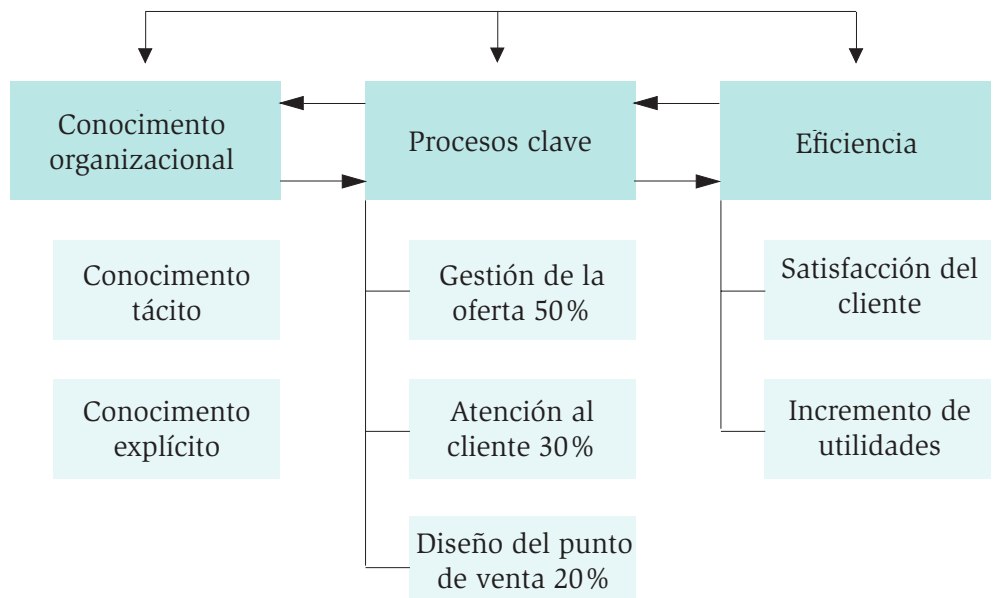

Fuente: Elaboración propia

Figura 5. Modelo de gestión del conocimiento para el comercio minorista

Cuadro 6. Valoración de las cualidades del modelo

\begin{tabular}{|lllcc|}
\hline EXPERTOS & CARÁCTER INTEGRADOR & VALOR TEÓRICO & VALOR METODOLÓGICO & $\begin{array}{c}\text { VALOR } \\
\text { PRÁCTICO }\end{array}$ \\
\hline 1 & 5 & 5 & 4 & 5 \\
\hline 2 & 5 & 5 & 4 & 5 \\
\hline 3 & 5 & 4 & 4 & 4 \\
\hline 4 & 4 & 4 & 4 & 5 \\
\hline 5 & 5 & 4 & 5 & 4 \\
\hline 7 & 5 & 5 & 4 & 5 \\
\hline 8 & 5 & 5 & 5 & 5 \\
\hline 9 & 5 & 4 & 5 & 5 \\
\hline Moda & 5 & 5 & 4 & 5 \\
\hline Media & 5 & 5 & 4 & 5 \\
\hline Mediana & 4.8888 & 4.5555 & 4.3333 & 4.7777 \\
\hline Desviación & 5 & 5 & 4 & 5 \\
\hline estándar & 0.3333 & 0.5270 & 0.5 & 0.4409 \\
\hline ISC & $98.3 \%$ & $97.3 \%$ & $97.5 \%$ & $97.7 \%$ \\
\hline
\end{tabular}

Fuente: Elaboraciòn propia. 
El Isc supera $85 \%$ en todos los casos, lo que se considera satisfactorio; según los resultados, se puede comprobar que es muy alta la valoración que le conceden los expertos al modelo propuesto y, según el criterio, el modelo queda validado.

\section{Consideraciones finales}

El conocimiento organizacional en el sector del comercio minorista de bienes puede clasificarse en tácito o explícito. Las habilidades y capacidades de las personas determinan la calidad del servicio en el punto de venta y la gestión de la oferta. Los procesos considerados clave en la gestión de empresas del sector son: gestión de la oferta, que garantiza que los bienes que el consumidor necesita se ofrezcan en el punto de venta; atención al cliente, que se refiere al nivel de relación y empatía con este en el momento de la compraventa, y diseño del punto de venta, que asegura el ambiente favorable para el cliente.

\section{FuENTES CONSULTADAS}

Barreiro Pousa, Luis (2002). "Enfoque estratégico de marketing para el comercio minorista de bienes en Cuba”. Tesis de doctorado en Ciencias Económicas. La Habana: Facultad de Economía-Universidad de La Habana.

Castro Díaz-Balart, Fidel (2002). Ciencia, innovación y futuro. Barcelona: Random House Mondadori.

García Morales, Víctor Jesús (2005). “Análisis de las barreras del aprendizaje organizacional desde la perspectiva de los centros educativos”. Dirección y Organización, 31, febrero, 46-65.

Marx, Carlos (1973). El Capital, t. II. La Habana: Editorial de Ciencias Sociales. Ollarves Levison, Yolibet, Mirla Estévez González y Luis Arturo Salguero (2012). "Propuesta para construcción de un modelo de gestión del conocimiento en una unidad de planificación y desarrollo de la upeL". Una Investigación, IV (8), 13-41.

Orozco Silva, Eduardo y Juan R. Carro Suárez (2002). “Propuesta de estrategia para la introducción de la gestión de la información y la gestión del 
conocimiento en las organizaciones cubanas". Ciencias de la Información, 33 (1), abril, 17-23.

Pérez Frías, Arturo e Ingrid E. Pérez R. (2004). “Conocimiento, gestión e innovación tecnológica como clave del rendimiento económico”. Revista de Ciencias Sociales, X (2), mayo-agosto, 338-349.

Ponjuán Dante, Gloria (2003). "Gestión documental, de información y del conocimiento... puntos de contacto y diferencias”. Ciencias de la Información, 34 (3), diciembre, 55-63.

------ (2006). Introducción a la gestión del conocimiento. La Habana: Félix Valera.

Pons García, Roberto, Zhao Hui Yuan y Dayana Duffus Miranda (2008). "El marketing y la imagen del comercio minorista de bienes". Contribuciones a la Economía [en línea]. Disponible en: http://www.eumed.net/ ce/2008b/gym.htm

Riesco González, Manuel (2006). El negocio es el conocimiento. Madrid: Díaz de Santos.

Saldarriaga Ríos, Juan Guillermo, Carlos Ariel Alzate Molina y Gustavo Alexander Gallego Álvarez (2012). Gestión del conocimiento [en línea]. Medellín: Biblioteca Digital Universidad de San Buenaventura. Disponible en: http://hdl.handle.net/10819/1058 [2013, 3 de julio].

Triana Cordoví, Juan, Ricardo Torres Pérez y Mariana Martín Fernández (2005). Cuba: hacia una economía basada en el conocimiento. La Habana: Editorial de Ciencias Sociales. 